\title{
Comparación de los factores de estructuración en la producción a partir de recortes generado en el desposte de cerdo
}

Comparison of the structuring factors in production from cuts generated in

the pig waste

\section{Comparação dos fatores estruturantes da produção a partir de cortes}

\section{gerados nos resíduos de suínos}

\author{
Álvarez Vallejo María Alejandra ${ }^{1}$ Rodríguez Prado Carlos Esteban ${ }^{1}$ y \\ Ladino Ospina María Cristina ${ }^{2}$ \\ ${ }^{11}$ ngenieros Agroindustriales, Universidad de los Llanos \\ ${ }^{2}$ Docente, Universidad de los Llanos \\ mcospina@unillanos.edu.co
}

Recibido 6 de junio 2019, Aceptado 30 de octubre 2019

\section{RESUMEN}

Con la intención de generar valor agregado a los recortes de carne de cerdo originados en el proceso de desposte, se utilizaron recortes del musculo de pierna de cerdo para elaborar carne reestructurada. Se tuvo en cuenta tres factores: tamaño de partícula, ligante y método de cocción. Se definió para el tamaño de partícula moler los recortes con un disco de $20 \mathrm{~mm}$ y cubicarlos con un tamaño de $1 \mathrm{~cm}^{3}$. Para el tipo de ligante se escogieron productos comerciales a base de carragenina y alginato de calcio al $1.5 \%$ y para la cocción, se utilizaron los métodos cocido y asado. Se eligió el diseño experimental factorial $2^{3}$ por polígonos ortogonales con 8 tratamientos y tres repeticiones. La comparación de medias de Tukey $(\alpha=0.05)$ se usó para realizar el análisis de varianza. Los resultados mostraron que no hubo diferencias significativas con respecto a la resistencia al corte entre los 8 tratamientos evaluados. Se evidenció que el tratamiento 5 (cubos, alginato, cocido) fue el que estuvo más cerca de presentar diferencias significativas con una resistencia al corte de $47.98 \mathrm{~N}$, siendo así, el que más se asemejaría a un corte de carne de musculo entero, pero con una característica adicional de tener una mayor terneza que genera un mayor valor agregado al producto final. 
Palabras clave: Ligantes, aglutinantes, recortes de carne, carne reestructurada.

\begin{abstract}
With the intention of generating added value to the cuts of pork originated in the preparation process. Pork leg muscle trimmings were used to make restructured meat. Three factors were taken into account: particle size, binder and firing method. It was defined for the particle size to grind the cutout with a disc of 20 millimeters and cube it with a size of $1 \mathrm{~cm}^{3}$. For the binder type the commercial products were selected from a base of carrageenan and a $1.5 \%$ calcium alginate and for cooking, there were used cooked methods and roasting. The experimental design chosen was $2^{3}$ by orthogonal polygons with eight treatments and three replicates. Comparison of Tukey mean $(\alpha=0.05)$ was used to perform the analysis of variance. The results show that there were no significant differences with respect to the cut resistance among the eight treatments evaluated. It was evidenced that the treatment 5 (cubes, alginate, cooked) was the closest to presenting significant differences with a cut resistance of $47.98 \mathrm{~N}$, being the one that most resembled a cut of meat of whole muscle but with an additional characteristic of tenderness that generates added value to the final product.
\end{abstract}

Keywords: Binders, meat cuts, restructured meat.

\title{
RESUMO
}

Com o intuito de gerar valor agregado aos cortes de carne de porco originários do processo de desmembramento, foram utilizados cortes no músculo da perna de porco para elaborar carne reestruturada. Três fatores foram levados em consideração: tamanho das partículas, aglutinante e método de cozimento. Foi definido para o tamanho da partícula moer as estacas com um disco de $20 \mathrm{~mm}$ e cobri-las com um tamanho de $1 \mathrm{~cm}^{3}$. Para o tipo de aglutinante, foram escolhidos produtos comerciais à base de carragena e alginato de cálcio a 1,5\% e, para 
cozinhar, foram utilizados os métodos cozidos e torrados. O delineamento fatorial experimental 23, por polígonos ortogonais, com 8 tratamentos e três repetições foi escolhido. A comparação da média de Tukey $(\alpha=0,05)$ foi utilizada para realizar a análise de variância. Os resultados mostraram que não houve diferenças significativas quanto à resistência ao corte entre os 8 tratamentos avaliados. Evidenciou-se que o tratamento 5 (cubos, alginato, cozido) foi o mais próximo de apresentar diferenças significativas com uma resistência ao corte de $47,98 \mathrm{~N}$, sendo o que mais se assemelhou a um corte de carne muscular inteira, mas com uma característica adicional de ter uma ternura que gera maior valor agregado ao produto final.

Palavras-chave: Fichários, cortes de carne, carne reestruturada.

\section{INTRODUCCIÓN}

Actualmente la industria cárnica ha venido incursionando en la creación de nuevos productos que satisfagan las necesidades del consumidor y que además generan un alto valor agregado para la industria. Algunos de estos avances de la industria están en la técnica de carne reestructurada.

Un producto reestructurado de carne es básicamente aquel que consiste de músculos enteros individualmente seleccionados, trozos de músculos o recortes de carne que pueden ser recombinados para elaborar un producto final (Kinsman et al., 1994). Existen algunos métodos de reestructuración de la carne y la mayoría se basan en la extracción de proteínas usando sal, fosfatos y manipulación mecánica. Posteriormente se aplica calor para formar una matriz de proteínas gelificadas. Estos productos deben ser comercializados precocidos o congelados porque las partículas de carne no se mantienen unidas cuando están crudas. La oxidación de lípidos, decoloración y baja aceptación de los consumidores son los principales problemas de los productos congelados. La carne reestructurada se puede ligar de dos formas, en calor o en frío. La carne ligada en calor forma geles por la interacción de las cadenas de miosina, ya que esta cuando se exponer al calor forma un gel 
irreversible que tiene mayor capacidad de retención de agua y propiedades elásticas fuertes. Para este proceso es necesario solubilizar la miosina durante el mezclado y antes del calentamiento, lo que facilita la formación de filamentos a altas temperaturas. Las altas temperaturas del proceso de cocción causan que la porción helicoidal de la proteína se desdoble y de manera aleatoria forme cadenas organizadas (Rocha, 2009).

La carne ligada en frío se utiliza para productos crudos y refrigerados. El ligado en frio también brinda a los procesadores la oportunidad de unir músculos de forma irregular. Muchos de estos productos pueden elaborarse combinando pedazos de carne o músculos enteros con aditivos y embutiéndolos en una funda y luego de un periodo corto de refrigeración, esta puede ser prensada en moldes y posteriormente rebanada. Algunos compuestos que se utilizan para ligar carnes en frío son los alginatos, los cuales se caracterizan por ser una goma hidrocoloide viscosa que es abundante en la pared celular de las algas café. En presencia de calcio, el alginato forma geles no reversibles. El compuesto fibrinógeno-trombina es un producto que es extraído del plasma de los bovinos. Cuando la enzima fibrinógeno reacciona con la trombina, otra enzima, se crea la fibrina. La fibrina tiene gran afinidad para ligar otras moléculas de fibrina, siempre y cuando el calcio esté presente.

Transglutaminasa: es industrialmente producida en cantidades comerciales por la fermentación de la levadura Streptomyces mobarensis o extraída de la sangre de los animales destinados a la alimentación humana y favorece el entrecruzamiento de las proteínas en varios procesos de la industria alimentaria.

Algunas marcas conocidas son FibrimexTM, Alginato [SMR] y ActivaTM. FibrimexTM (FIBRIMEX, 2004). Se basa en el principio de coagulación de la sangre en el cual el fibrinógeno es activado por la enzima trombina para formar la fibrina que puede ser usada como gel natural en la reestructuración de carne. El ácido algínico es una goma viscosa que es usada junto con alguna fuente de calcio como agente gelificante. ActivaTM tiene como componente activo la enzima transglutaminasa que puede catalizar la formación de uniones entre proteínas (Grifinm, 2002). Fibrimex es el único de los anteriores productos aprobado en 
Canadá, mientras que Activa, alginatos y Fibrimex son aprobados por Japón y los Estados Unidos de Norteamérica (Vigo, 2009).

El presente estudio se realizó a partir de la necesidad de crear nuevos productos que generen valor agregado a la cadena cárnica porcícola, debido a que el consumo de la carne de cerdo en Colombia en los últimos años ha venido incrementando, según las cifras de beneficio de porcinos para el año 2015, se sacrificaron 386.685.836 cabezas de ganado porcino, aumentando en 447.802 cabezas frente al año inmediatamente anterior, lo cual correspondió a una variación del 13.8\% (PORCICOL, 2015).

Mediante el proceso de reestructuración, un método que consiste en reducir los cortes cárnicos (en este caso recortes) y luego mezclarlo con sales y productos aglutinantes para lograr extraer la proteína, empacarlo al vacío para generar productos similares a las de las piezas íntegras, además, de mejorar características de los productos como la textura, contenido de grasa y la forma; la industria tiene nuevas alternativas, que combinadas con la innovación, desarrolla nuevos productos que permiten la comercialización de los subproductos o recortes, dándoles un valor agregado y dejando a la industria una mayor rentabilidad. Dentro de los métodos de reestructuración, existen diferentes productos aglutinantes que proporcionan características al producto final de acuerdo a su naturaleza y sus propiedades. El producto comercial a base de carragenina como ligante, se caracteriza por su resistencia a temperaturas de fritura $\left(150^{\circ} \mathrm{C}\right)$ permitiendo ligar significativamente agua a partir de $\operatorname{los} 46^{\circ} \mathrm{C}$, mientras que las proteínas de la carne inician su coagulación a los $52^{\circ} \mathrm{C}$, aumentando así eficientemente la retención de agua durante la cocción y mejorando la firmeza, el tajado y la palatabilidad. Por otra parte, los productos comerciales a base de Alginato de Calcio actúan como gelificantes, esta propiedad está determinada por su masa molecular, entre mayor sea la masa, mayores son las capacidades viscosificantes (Aguayo et al., 2017); de esta forma en la reestructuración de productos cárnicos el alginato como aglutinante ayuda a unir los trozos de carne y disminuye la concentración de Cloruro de Sodio 
a utilizar, previniendo la decoloración del producto final, evitando así el rechazo de los consumidores.

La reestructuración de la carne tiene como objetivo imitar la apariencia y los atributos de un producto de tejido entero, proporcionando ventajas adicionales como la modificación de los componentes químicos del musculo por medio de la aplicación de tratamientos; así mismo la formulación de los productos por la adición de ingredientes deseados, que pueden mejorar las propiedades fisicoquímicas de los cortes, además de enriquecer y hacer más disponibles algunos compuestos que pueden resultar beneficiosos para la salud humana.

En el presente estudio se quiso evaluar los factores de estructuración que permitan obtener un corte reestructurado con propiedades similares a las de la carne de cerdo entera. Para esto se evaluaron dos tipos de ligantes químicos que podría utilizarse en la elaboración de carne reestructura a concentraciones máximas permitidas por la ley. A su vez evaluando el tamaño de partícula a condiciones de presión, tiempo y temperatura constantes, comparando el comportamiento de los aglutinantes frente a dos métodos de cocción de un corte reestructurado.

\section{METODOLOGÍA}

En este capítulo se detallan las diferentes actividades ejecutadas para el desarrollo de este trabajo. El experimento se realizó en una empresa comercializadora y en el laboratorio de Análisis Sensorial de la Universidad Autónoma Chapingo, México D.F. El diseño experimental que se planteó es un diseño factorial $2^{3}$ por polígonos ortogonales, basado en lo descrito por Pulido, (2008) Estos son especialmente útiles para investigar la forma como influyen $k$ factores sobre una variable respuesta, y en cada factor se consideran dos niveles solamente. Es común analizarlos como contrastes ortogonales para estimar efectos principales e interacciónales. 
Modelo estadístico:

$\gamma_{i j k l}=\mu+A_{i}+B_{j}+C_{k}+(A B)_{i j}+(A C)_{i k}+(B C)_{j k}+(A B C)_{i j k}+e_{I(i j k)}$.

$\gamma_{i j k l}=$ Variable de respuesta

$\mu=$ Media general

$A_{i}=$ Efecto del tamaño de partícula en su nivel i

$B_{j}=$ efecto del ligante en su nivel $\mathrm{j}$

$C_{k}=$ Efecto del método de cocción en su nivel $\mathrm{k}$

$(A B)_{i k}=$ Efecto de la interacción $\mathrm{AB}$ al nivel i,k

$(A B C)_{i j k}=$ Efecto de la interacción $\mathrm{ABC}$ al nivel i, j, $\mathrm{k}$

$(i j k)=$ Error aleatorio

Los factores para este experimento fueron los aglutinantes, el tamaño de partícula y el método de cocción. Los niveles para los aglutinantes, fueron las pruebas con el ligante WBS 203 de la marca comercial Wenda Ingredients en cuya fórmula se incluye el Alginato de Sodio y el ligante Gelybekam 20-10 Bi de la marca Bekarem que incluye como ingrediente la Carragenina, los niveles para el tamaño de partícula fueron cubos y molida en el disco, y los niveles del factor método de cocción serán cocido (Figura 1) y asado (Figura 2).

La variable de respuesta será la fuerza de corte y se realizarán tres (3) réplicas para cada experimento, cada unidad de estudio tendrá medidas rectangulares de dimensión $2.5 \mathrm{~cm} \times 2.5 \mathrm{~cm}$ de lado y $1 \mathrm{~cm}$ de longitud y serán necesarias 24 unidades de estudio, que serán comparadas con músculo de pierna cerdo. E análisis de los datos se realizó con el Software de análisis estadístico SAS y para la comparación de medias de los tratamientos se utilizó la prueba de Tukey $(P<0.05)$.

Los tratamientos usados fueron los siguientes: 1) Carne molida, con el ligante alginato de sodio (LA-Na) y método de cocción; cocido. 2) Carne molida, con LA-Na y método de cocción: asado. 3) Carne molida, con el ligante carragenina (LC) y método de cocción: cocido. 4) Carne molida, con LA y el método de cocción: asado. 
5) Carne en cubos, con LA-Na y método de cocción: cocido. 6) Carne en cubos, con LA-Na y método de cocción: asado. 7) Carne en cubos, con LA y método de cocción: cocido. 8) Carne en cubos, con LA y método de cocción: asado.

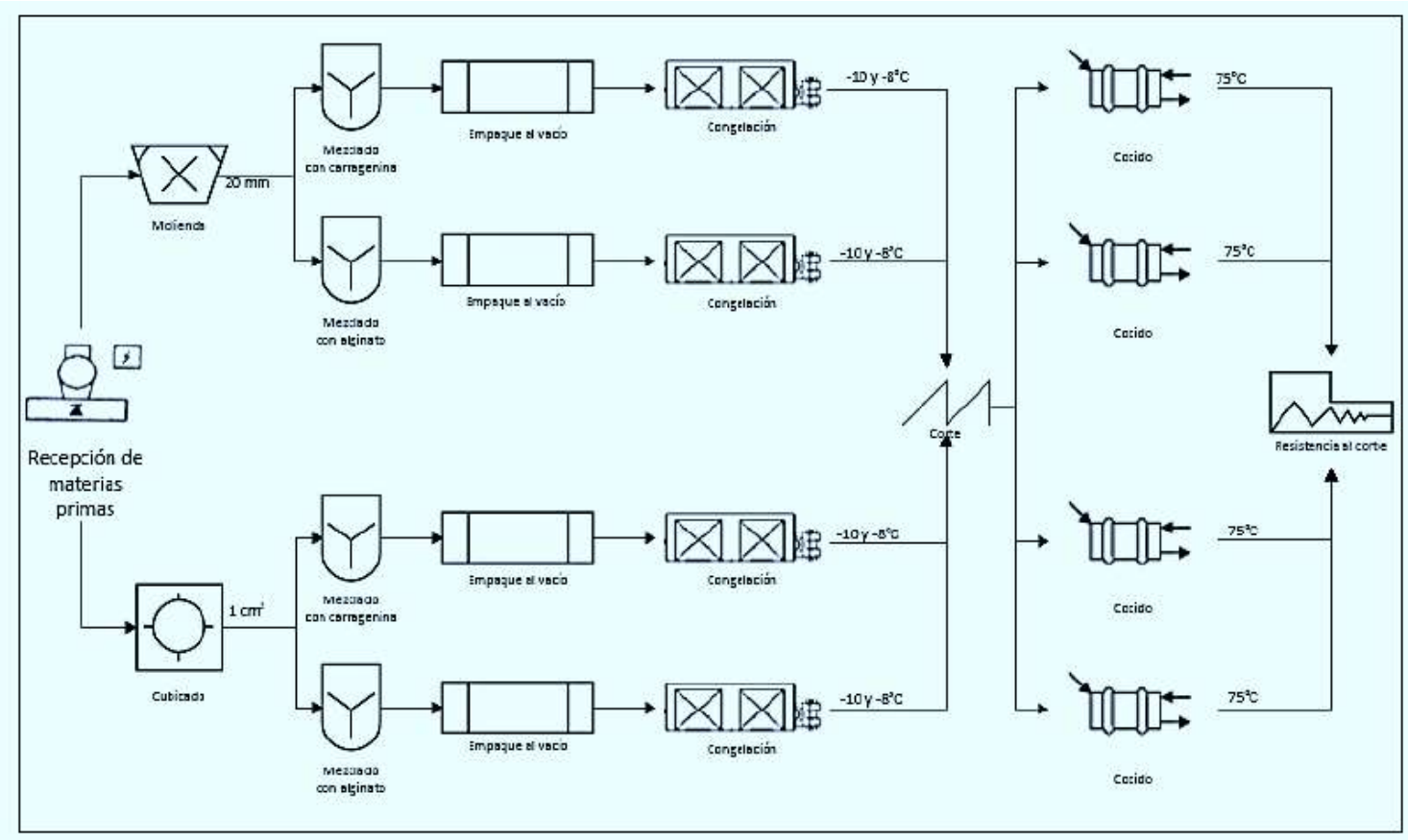

Figura 1. Diagrama de proceso para el método de cocción cocido

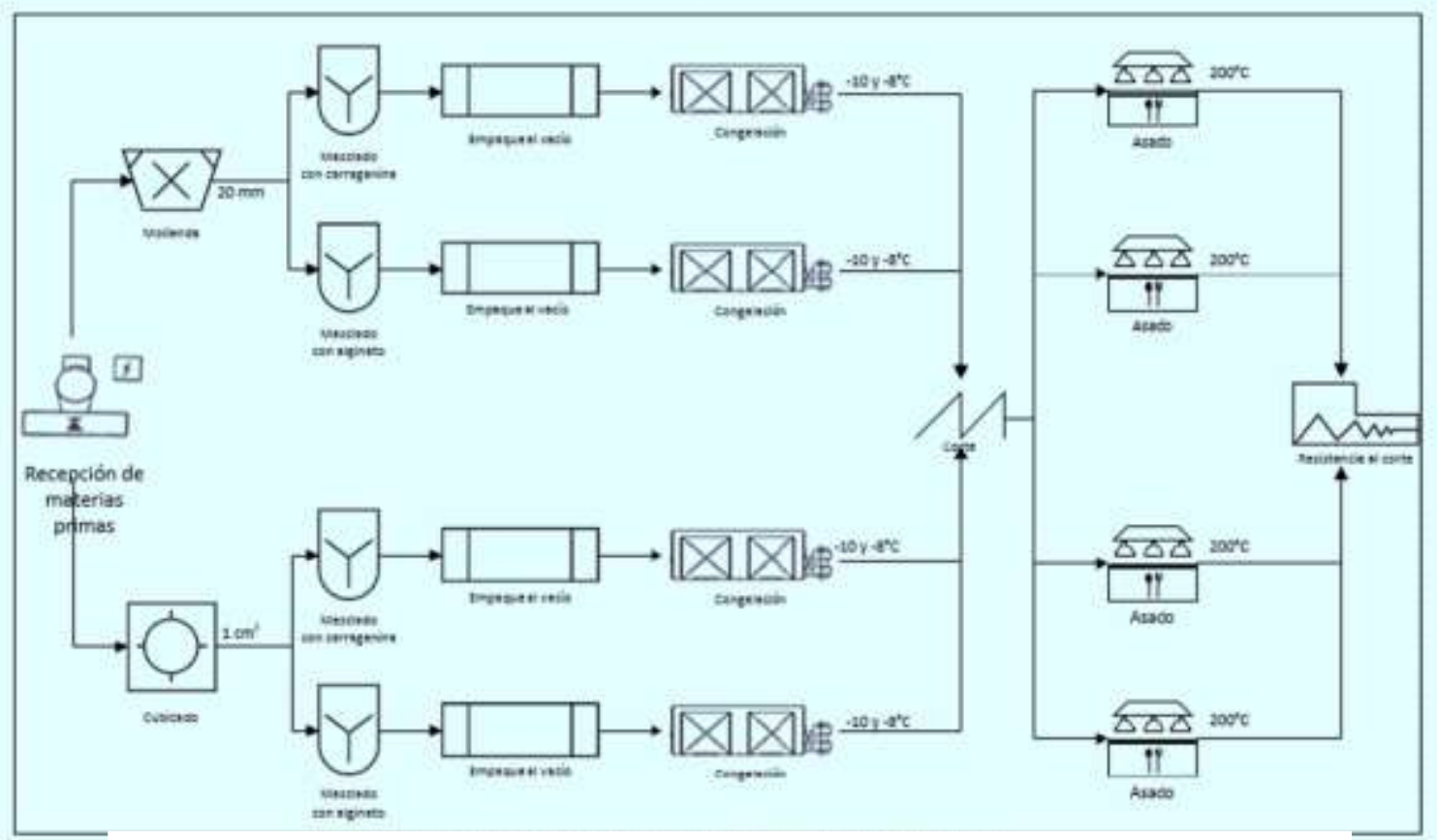

Figura 2. Diagrama de proceso para el método de cocción asado 
Para el desarrollo de la metodología de preparación del producto se tuvo en cuenta la tesis: "Desarrollo de un producto reestructurado a partir de carne de res de bajo valor comercial" Presentada por Álvaro Salinas Roca y el capítulo XVIII Tecnología de carnes reestructuradas para agregar valor a carnes de baja calidad y aceptabilidad escrito por Márquez et al., (2008) publicado en el libro Ganadería Mestiza de Doble Propósito. Las materias primas utilizadas fueron: la carne como ingrediente principal y como aditivos el agua y los aglutinantes que son WBS 203 (alginato de sodio) y el Gelybekam (carragenina).

\section{RESULTADOS Y DISCUSIÓN}

Los contrastes entre los valores de los diferentes efectos, siendo el efecto a, el contraste entre el tamaño de partícula; el efecto b, el contraste entre ligantes; el efecto c, el contraste entre métodos de cocción; el efecto ab, el contraste entre tamaño de partícula y ligantes; el efecto ac, el contraste entre tamaño de partícula y métodos de cocción; el efecto bc, el contraste entre ligantes y métodos de cocción; el efecto ab, el contraste entre tamaño de partícula, ligantes y métodos de cocción. (Tabla1).

Tabla 1. Contrastes entre efectos

\begin{tabular}{cccccccc}
\hline Contraste & $\begin{array}{c}\text { Efecto } \\
\mathbf{a}\end{array}$ & $\begin{array}{c}\text { Efecto } \\
\text { b }\end{array}$ & $\begin{array}{c}\text { Efecto } \\
\text { c }\end{array}$ & $\begin{array}{c}\text { Efecto } \\
\text { ab }\end{array}$ & $\begin{array}{c}\text { Efecto } \\
\text { ac }\end{array}$ & $\begin{array}{c}\text { Efecto } \\
\text { bc }\end{array}$ & Efecto abc \\
\hline $\operatorname{Pr}<0.05$ & 0.87 & 0.883 & 0.066 & 0.961 & 0.684 & 0.527 & 0.837 \\
\hline
\end{tabular}

Fuente: Los autores

No se observó ningún efecto significativo en los diferentes tipos de ligantes, métodos de cocción y tamaños de partícula utilizados no influyen en la fuerza de corte de los diferentes tratamientos. Por otra parte, el contraste que más se acerca a un $\mathrm{P}<0.05$ es el efecto $\mathrm{c}$, es decir, que los métodos de cocción asado y cocido, interfieren más en la fuerza de corte (Tabla 1). 
El agrupamiento de acuerdo a la prueba de comparación múltiple de Tukey, indica que las medias con la misma letra no son significativamente diferentes. Sin embargo, las medias de los tratamientos 5, 1, 7 y 3 son las más altas que van desde 47.98 hasta 39.00 y los tratamientos $4,2,8$ y 6 tienen las medias con los valores más bajos que van desde 32.93 a 23.66, esto demuestra que hay una separación entre el factor c (método de cocción), es decir entre los tratamientos con carne cocida y carne asada (Tabla 2).

La carne cocida tuvo una temperatura de $75^{\circ} \mathrm{C}$ y en los tratamientos térmicos suaves (temperaturas hasta $100^{\circ} \mathrm{C}$ o ligeramente superiores) se rompen uniones de baja energía, como puentes hidrógeno (Lupano, 2013). Es decir, que las bajas temperaturas no hidrolizan las proteínas, por lo tanto, la carne presenta una mayor resistencia al corte.

Tabla 2. Comparación de medias Tukey

\begin{tabular}{ccccccccc}
\hline Tratamiento & $\mathbf{5}$ & $\mathbf{1}$ & $\mathbf{7}$ & $\mathbf{3}$ & $\mathbf{4}$ & $\mathbf{2}$ & $\mathbf{8}$ & $\mathbf{6}$ \\
\hline Media (N) & 47.98 & 47.21 & 42.52 & 39 & 32.93 & 26.43 & 25.71 & 23.66 \\
$\mathrm{~N}$ & 3 & 3 & 3 & 3 & 3 & 3 & 3 & 3 \\
$\begin{array}{c}\text { Agrupamiento } \\
\text { de Tukey }\end{array}$ & $\mathrm{A}$ & $\mathrm{A}$ & $\mathrm{A}$ & $\mathrm{A}$ & $\mathrm{A}$ & $\mathrm{A}$ & $\mathrm{A}$ & $\mathrm{A}$ \\
\hline
\end{tabular}

Fuente: Los autores

Por otra parte, de acuerdo a lo reportado por Lupano, (2013) la cocción a temperaturas superiores a los $200^{\circ} \mathrm{C}$, como es el caso de la superficie de la carne cocida a la parrilla o a la plancha, producen una hidrólisis de los enlaces peptídicos y la isomerización de residuos aminoácidos; haciendo la carne más digestible y con una menor resistencia al corte.

Los valores testigos (tratamientos 9 y 10) fueron medidos en musculo de pierna de cerdo entero con el proceso de cocción cocido y asado respectivamente. En relación a la fuerza de corte de los tratamientos testigo, la media fue más alta para los tratamientos con método de cocción cocidos (85.79) que para los asados (68.17), y en general, los tratamientos (reestructurados) presentaron una fuerza de corte menor que la de los testigos (carne entera), lo cual indica, que mediante el proceso 
de reestructuración se pueden obtener cortes más blandos. Por otra parte, en un estudio realizado por Salinas, (2007) se hizo un análisis sensorial de aceptación y preferencia para comparar la carne reestructurada con un bistec del músculo tríceps brachii y los panelistas prefirieron la apariencia del bistec y el sabor de la carne reestructurada, aunque no hubo diferencias en suavidad, jugosidad y aceptación general. La preferencia fue igual para el bistec y para la carne reestructurada (Salinas, 2007).

\section{CONCLUSIONES}

Mediante la reestructuración cárnica se puede lograr que, a partir de la carne de animales adultos con una alta resistencia al corte, y que normalmente se utiliza para la producción de embutidos de tipo económico, se elabore en un producto reestructurado, haciendo que esta sea más tierna para los consumidores, y que los productores obtengan mayores ganancias.

Según las pruebas desarrolladas, no existe diferencia significativa en la utilización de un ligante comercial en cuya fórmula se incluye el alginato de sodio o la carragenina. Por lo tanto, es indiferente el uso de cualquiera de los dos ligantes en la elaboración de un producto cárnico reestructurado.

Se evidenció que no hubo diferencias significativas entre los dos tamaños de partícula evaluados ante la variable de respuesta utilizada, en este caso la resistencia al corte. Por lo cual, se concluye que estos dos tamaños de partícula son viables para el desarrollo de un producto cárnico reestructurado.

Se evidenció que el método de cocción tampoco tuvo diferencias estadísticas significativas entre los tratamientos. No obstante, entre aquellos que fueron cocidos y los que fueron asados, se observaron diferencias en cuanto a la resistencia al corte ya que los que fueron cocidos a más alta temperatura presentaron una fuerza de corte menor. Se concluye así, que a mayor temperatura de cocción la resistencia al corte es menor y a menor temperatura, la resistencia es mayor. 


\section{RECOMENDACIONES}

La evaluación de la fuerza de corte, se tomó como una variable de respuesta, por lo que resultaría interesante evaluar otras variables como elasticidad, cohesividad y realizar pruebas organolépticas.

Para futuros estudios en carne reestructurada es importante tener en cuenta el uso de ligantes enzimáticos como la transglutaminasa. Otro aspecto a tener en cuenta es la utilización de métodos de cocción como el horneado.

Para la fabricación de este producto a nivel industrial para su comercialización, es recomendable utilizar algunos aditivos como el tripolifosfato de sodio, para mejorar la cohesividad.

\section{REFERENCIAS BIBLIOGRÁFICAS}

1. Aguayo, R.; Contreras, M.; Hernández, B.; Vázquez M. Gelificación de alginatos. Centro Universitario Anglo Mexicano de Morelos, S.C. 2017. [online] Recuperado $24 \quad$ Febrero 2017. Disponible En: http://www.acmor.org.mx/sites/default/files/223.\%20Alginatos.pdf

2. Asociación Colombiana de porcicultores. Como le fue a la porcicultura colombiana en 2015, expectativas para el 2016. Ed.209 enero-febrero 2016. [online] Recuperado 02 Enero 2017. Disponible En: http://www.porcicol.org.co/porcicultores/images/porcicultores/revistas/209.pdf

3. FIBRIMEX. What is fibrimex? 2004. [online] Recuperado 15 Mayo 2016. Disponible En: http://www.fibrimex.com/index.asp

4. Grifinm M, Casadio R, Bergamini C. Tranglutaminasas: el pegante biológico de la naturaleza. Biochemistry Journal, dic: 77-96. 2002.

5. Lupano, E. Modificaciones de componentes de los alimentos: cambios químicos y bioquímicos por procesamiento y almacenamiento. Buenos Aires: Editorial de la Universidad de la Plata., 218 p. 2013.

6. Marquez E. Estabilidad de productos cárnicos reestructurados crudos con agregado de transglutaminasa y plasma de bovino. Revista Científica Maracaibo, 5 (18). 2008.

7. Pulido G, H. Análisis y diseño de experimentos. Segunda edición. McGraw Hill. 2008.

8. Rocha, A. Fabricación de productos reestructurados. Tecnología de Procesamiento. CarneTec. 2009. 
9. Salinas, Á. Desarrollo de un producto reestructurado a partir de carne de res de bajo valor comercial. Zamorano. Trabajo de grado Ingeniería en Agroindustria Alimentaria, Universidad de Zamorano. 2007.

10. Vigo, C. Características fisicoquímicas de un reestructurado de carne de alpaca con inclusión de pecana y transglutaminasa. Lima, Perú. Trabajo de grado (Médico Veterinario). Universidad Nacional Mayor de San Marcos. 96 p. 2014. 